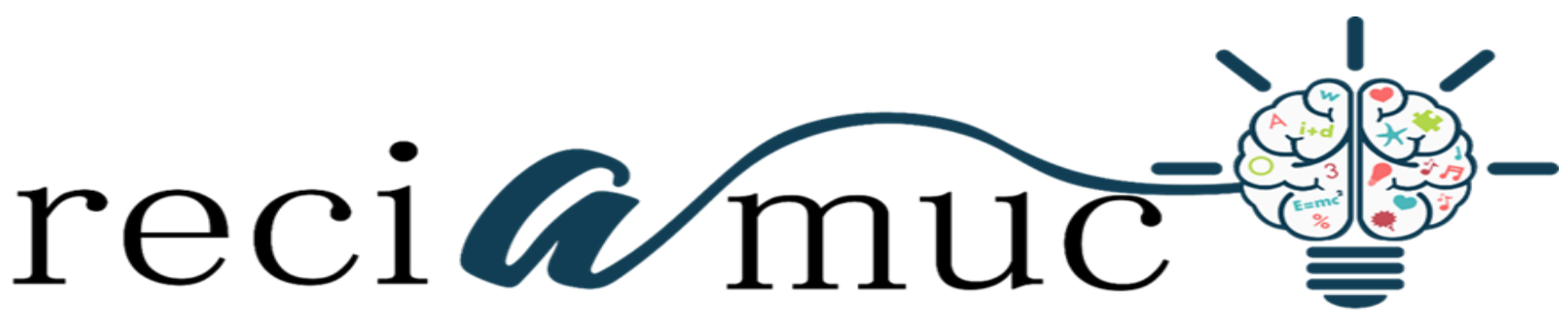

Revista cientifica de investigación actualización del mundo de las ciencias

\author{
Victoria Samanta Luna Panimboza a ${ }^{\text {a }}$ Frowen Miguel Zambrano Solorzano ${ }^{\text {b }}$, Ligia \\ Elena Panezo Carbo ${ }^{c}$; Julian Alfonso Luna Montalván ${ }^{d}$
}

Uso terapéutico de la moringa oleífera en pacientes diabéticos

Therapeutic use of moringa oleifera in diabetic patients

Revista Científica de Investigación actualización del mundo de las Ciencias. Vol. 3 núm., 3, julio, ISSN: 2588-0748, 2018, pp. 724-737

DOI: $10.26820 /$ reciamuc/3.(3).julio.2019.724-737

URL: http://reciamuc.com/index.php/RECIAMUC/article/view/300

Código UNESCO: 3210 Medicina Preventiva

Tipo de Investigación: Artículo de Revisión

() RECIAMUC; Editorial Saberes del Conocimiento, 2019

Recibido: 28/04/2019

Aceptado: 19/05/2019

Publicado: 01/07/2019

Correspondencia: victoria_luna19@outlook.es
a. Médico; Investigador Independiente; Guayaquil, Ecuador; victoria_luna19@ outlook.es
b. Médico; Investigador Independiente; Guayaquil, Ecuador; drfroz@ hotmail.com
c. Médico; Investigador Independiente; Guayaquil, Ecuador; queen_black85@hotmail.com
d. Médico; Investigador Independiente; Guayaquil, Ecuador; d.rjluna@ hotmail.com 


\section{Uso terapéutico de la moringa oleífera en pacientes diabéticos}

Vol. 3, núm. 3., (2019)

Victoria Samanta Luna Panimboza; Frowen Miguel Zambrano Solorzano; Ligia Elena Panezo

Carbo; Julian Alfonso Luna Montalván

\section{RESUMEN}

La Moringa Oleífera es una planta usada en muchas partes del mundo por sus múltiples beneficios entre ellos su potencial medicinal para tratar diversas enfermedades, entre ellas la diabetes. La presente investigación plasma algunos estudios clínicos y preclínicos que evidencian el uso de esta planta en el tratamiento de la diabetes. Para su desarrollo se llevó a cabo una recopilación y revisión de material documental bibliográfico digital. De los estudios revisados se observó que, con diferentes partes de la planta y formas de preparación y administración, tanto en animales como en humanos, se obtuvo un resultado hipoglucemiante. Asimismo, se observó un aumento de peso en los sujetos de estudio, no obstante, no se presentó ningún efecto tóxico. En conclusión, se evidencia que el uso de la Moringa Oleífera disminuye los niveles de glucosa en sangre, además del colesterol y triglicéridos, por ser un potente antioxidante, además de que no presenta efectos adversos. Todos los estudios coinciden en la falta de nuevas investigaciones que respalden estos hallazgos y se enfoquen en el contenido de los principios activos de la planta que ocasionan tales resultados, además de la precisión en la dosificación y los efectos a largo plazo.

Palabras Claves: Moringa; Oleífera; Terapia; Pacientes; Diabetes. 


\title{
Uso terapéutico de la moringa oleífera en pacientes diabéticos
}

Vol. 3, núm. 3., (2019)

Victoria Samanta Luna Panimboza; Frowen Miguel Zambrano Solorzano; Ligia Elena Panezo Carbo; Julian Alfonso Luna Montalván

\begin{abstract}
Moringa Oleifera is a plant used in many parts of the world for its multiple benefits, including its medicinal potential to treat various diseases, including diabetes. The present investigation shows some clinical and preclinical studies that show the use of this plant in the treatment of diabetes. For its development, a compilation and review of digital bibliographic documentary material was carried out. From the reviewed studies it was observed that, with different parts of the plant and forms of preparation and administration, both in animals and in humans, a hypoglycaemic result was obtained. Also, an increase in weight was observed in the study subjects, however, no toxic effect was present. In conclusion, it is evident that the use of Moringa Oleifera decreases blood glucose levels, in addition to cholesterol and triglycerides, as it is a potent antioxidant, in addition to having no adverse effects. All the studies coincide in the lack of new research to support these findings and focus on the content of the active principles of the plant that cause such results, in addition to the precision in the dosage and the long-term effects.
\end{abstract}

Key Words: Moringa; Oleifera; Therapy; Patients; Diabetes. 


\section{Uso terapéutico de la moringa oleífera en pacientes diabéticos}

Vol. 3, núm. 3., (2019)

Victoria Samanta Luna Panimboza; Frowen Miguel Zambrano Solorzano; Ligia Elena Panezo

Carbo; Julian Alfonso Luna Montalván

\section{Introducción.}

Es innegable la importancia de las plantas medicinales en todo el mundo por cuanto constituyen una fuente importante de agentes terapéuticos potenciales en el combate de diversas enfermedades, representan una fuente curativa natural y accesible por su bajo costo, sobre todo en países de bajos ingresos.

Esta práctica del uso de plantas medicinales se considera parte de la medicina complementaria y/o alternativa, entendida estas como "conjunto de sistemas, prácticas y productos que, en general, no se consideran parte de la medicina convencional", la cual ha venido tomando parte en el combate contra las enfermedades y la conservación de la buena salud. Cada día son menores los límites que separan a la medicina complementaria y alternativa de la convencional, lo que hace presumir que con el transcurrir del tiempo, ciertas prácticas de medicina complementaria y alternativa podrían pasar a ser aceptadas de forma general. (Centro Nacional de Medicina Complementaria y Alternativa de los Estados Unidos, 2011)

Los tratamientos estándar (medicina convencional), se basan en resultados de investigaciones científicas y, por ende, son aceptados y usados ampliamente. La mayoría de los tipos de medicina alternativa por el contrario carece de investigaciones científica, ésta incluye regímenes de alimentación especiales, preparados con hierbas, megadosis de vitaminas, tés especiales, terapia con imanes, acupuntura, entre otros. (Instituto Nacional del Cáncer de los Estados Unidos, 2019) 


\section{Uso terapéutico de la moringa oleífera en pacientes diabéticos}

Vol. 3, núm. 3., (2019)

Victoria Samanta Luna Panimboza; Frowen Miguel Zambrano Solorzano; Ligia Elena Panezo Carbo; Julian Alfonso Luna Montalván

La Moringa es una planta con múltiples usos conocidos en la medicina alternativa o convencional, combate la desnutrición al ser un multivitamínico potente, la contaminación ambiental, se usa en el tratamiento de múltiples enfermedades, entre ellas la diabetes.

La Organización Mundial de la Salud, (2016) en su informe mundial sobre la diabetes, define esta enfermedad de tipo crónica grave, como aquella desencadenada por la falta de producción de insulina por parte del páncreas (una hormona que regula el nivel de azúcar, o glucosa, en la sangre), o en aquellos casos donde el organismo no es capaz de utilizar con eficacia la insulina que produce. Esta enfermedad representa un "importante problema de salud pública y una de las cuatro enfermedades no transmisibles (ENT) seleccionadas por los dirigentes mundiales para intervenir con carácter prioritario”. (p. 1)

En 1980 el número de personas afectadas por la diabetes era de 108 millones, esta cantidad sufrió un gran aumento para 2014 de 422 millones. La prevalencia mundial de la diabetes en adultos fue de $8,5 \%$ en 2014 y su aumentado se presenta con mayor rapidez en los países de ingresos medianos y bajos. La diabetes causa ceguera, infarto de miocardio, insuficiencia renal, accidente cerebrovascular, amputación de los miembros inferiores, entre otros. En 2015 se estima que la diabetes fue la causa directa de 1,6 millones de muertes. "Según proyecciones de la OMS, la diabetes será la séptima causa de mortalidad en 2030". (Organización Mundial de la Salud, 2018)

La medicina no convencional, ya sea usada en vez de la convencional o complementaria, representa en este sentido una nueva alternativa para la prevención y el control de la diabetes. De forma económica, segura y al alcance de los países de bajos recursos o sub desarrollados. 


\section{Uso terapéutico de la moringa oleífera en pacientes diabéticos}

Vol. 3, núm. 3., (2019)

Victoria Samanta Luna Panimboza; Frowen Miguel Zambrano Solorzano; Ligia Elena Panezo

Carbo; Julian Alfonso Luna Montalván

Para Martín, y otros, (2013) a pesar de la falta de evidencia científica, el amplio consumo humano de Moringa Oleífera como dieta y remedio terapéutico durante siglos, sin reporte de casos de alergias y toxicidad, podría significar un aval suficiente de su inocuidad. No obstante, esta razón no bastaría si no estuviese respaldado por evidencias científicas. La mayoría de estas pruebas confirman un elevado margen de seguridad de los extractos de semillas y otras partes de esta planta, por lo que se podría afirmar que la no toxicidad de sus semillas se encuentra científicamente confirmada. (p. 143)

La presente revisión actualizada está orientada a plasmar algunos estudios, tanto preclínicos como clínicos, que evidencian los beneficios del tratamiento con Moringa Oleífera en el control de la diabetes.

\section{Materiales y Métodos.}

El presente estudio se realizó mediante una recopilación y revisión de material documental bibliográfico digital el cual fue utilizado para documentar y plasmar algunas evidencias científicas importantes que respaldan el uso de la Moringa Oleífera en el control de la diabetes. Se revisaron algunos artículos (ensayos preclínicos y clínicos) elaborados entre el año 2011 y 2019 donde se analizaron los efectos de diversas partes de la planta de moringa oleífera en la diabetes, con énfasis en su efecto hipoglucemiante y la toxicidad que pudiera causar dicho tratamiento.

En este orden de ideas, para Ríos, (2017) la investigación documental “...recurre a la consulta de documentos para obtener sus resultados". (p. 81). 


\section{Uso terapéutico de la moringa oleífera en pacientes diabéticos}

Vol. 3, núm. 3., (2019)

Victoria Samanta Luna Panimboza; Frowen Miguel Zambrano Solorzano; Ligia Elena Panezo Carbo; Julian Alfonso Luna Montalván

En razón de lo cual, el presente estudio se trata de una investigación documental bibliográfico dado el tipo de fuente usada para su elaboración.

\section{Resultados.}

La Moringa es un género de arbustos y árboles con innumerables usos: hojas, raíces y vainas no maduras se consumen como hortaliza. Todas sus partes son comestibles: la corteza, vainas, nueces, hojas, semillas, raíces, tubérculos y flores. "Los productos de Moringa tienen propiedades antibióticas, contra el tripanosoma y la hipotensión, antiespasmódicas, antiulcerosas, antiinflamatorias, hipocolesterolémicas e hipoglucémicas". (Organización de las Naciones Unidas para la Alimentación y la Agricultura, 2019)

La Moringa Oleífera es la especie más popular de la Moringa y en la medicina tradicional india, es usada en el tratamiento de la diabetes y para la hipertensión arterial. En las naciones africanas popularmente se han reportado casos de curaciones milagrosas de diabetes e hipertensión atribuidas al uso de remedios elaborados a partir de esta planta. A pesar de que faltan estudios que garanticen su mejor uso e inocuidad, son muchos los avances en materia de evidencias convincentes que se han obtenido hasta ahora. Algunos fitoquímicos contenidos en las hojas y los frutos de Moringa Oleífera han revelado su potencial para el control de la diabetes, además de su alto contenido de vitaminas, característica esencial para el uso en la terapia de esta enfermedad. Además de esto, la vitamina D resulta fundamental para el adecuado funcionamiento del páncreas y la secreción de insulina. Por otra parte, la presencia de $\beta$-caroteno disminuye el riesgo de ceguera en pacientes diabéticos. Asimismo, la vitamina B-12 presenta beneficios en el tratamiento de la neuropatía diabética. Por último, previene el desarrollo de 


\section{Uso terapéutico de la moringa oleífera en pacientes diabéticos}

Vol. 3, núm. 3., (2019)

Victoria Samanta Luna Panimboza; Frowen Miguel Zambrano Solorzano; Ligia Elena Panezo

Carbo; Julian Alfonso Luna Montalván

complicaciones diabéticas como las cataratas, por su contenido de vitamina C. (Martín, y otros, 2013, p. 142,143)

Estudios pre clínicos de la Moringa Oleífera en el control de la diabetes

Villarruel, y otros, (2018) realizaron un estudio preclínico en ratas diabéticas con la finalidad de demostrar el uso terapéutico de las hojas de Moringa Oleífera motivado a su posible capacidad para disminuir la glucosa en sangre. Se usó el extracto de hoja de la planta, a objeto de conocer los efectos sobre la toxicidad de la glucosa, el colesterol, los triglicéridos, el peso corporal, entre otros. Se obtuvo como resultado que el consumo de las hojas "mostró un efecto hipoglucémico, no obstante, en el peso corporal mostró un aumento. Ante esta evidencia los autores concluyeron que la Moringa Oleífera tiene un efecto hipoglucémico, sin embargo, se requieren más estudios que garanticen este mecanismo de acción. (p. 8)

Por otra parte, Al-Malki \& El Rabey, (2015) investigaron la actividad antidiabética de dos dosis bajas de polvo de semilla de Moringa (50 y $100 \mathrm{mg} / \mathrm{kg}$ de peso corporal, en la dieta) en ratas macho con diabetes inducida por estreptozotocina, donde concluyeron que con las bajas dosis de Moringa se reveló una excelente actividad antidiabética por su contenido de antioxidantes, glucomoringina, fenoles y flavonoides. Además, las dosis más bajas de Moringa en estudio presentan mayores beneficios médicos cuando son usadas como complemento alimenticio en la dieta de pacientes con diabetes. (p. 11)

Onyagbodor \& Aprioku, (2017) investigaron el potencial protector y mejorador del extracto de etanol de Hoja de Moringa oleífera (50-200 mg / kg) en la diabetes inducida por alloxan en ratas. "Los resultados demuestran que la hoja de Moringa Oleífera posee potenciales 


\section{Uso terapéutico de la moringa oleífera en pacientes diabéticos}

Vol. 3, núm. 3., (2019)

Victoria Samanta Luna Panimboza; Frowen Miguel Zambrano Solorzano; Ligia Elena Panezo Carbo; Julian Alfonso Luna Montalván

antidiabéticos protectores y de mejora en ratas. Lo cual puede ser debido a su actividad antioxidante". (p. 10)

Por otra parte, Edoga, Njoku, Amadi, \& Okeke, (2013) llevaron a cabo un estudio para demostrar los efectos de Moringa oleífera lam sobre el azúcar en la sangre de ratas albinas, los cuales de los resultados obtenidos concluyeron que el tratamiento con Moringa oleífera es comparable con el fármaco de referencia tolbutamida. Se pueden realizar más estudios para identificar los principios activos que ocasionaron los resultados, principios responsables del efecto hipoglucemiante. (p. 89)

Nadro, Audu, \& Glen, (2018) estudiaron los posibles efectos terapéuticos de la Moringa Oleífera en el extracto acuoso y el aceite de la semilla sobre determinados marcadores bioquímicos en la diabetes mellitus, enfermedad inducida en una población de 35 ratas albinas por estreptozotocina. Los resultados demostraron que la semilla de Moringa oleífera tiene un efecto anti hiperglucémico y, en consecuencia, puede aliviar el hígado y los daños generales asociados a la diabetes mellitus, como el mejoramiento de la función renal y la función hepática. Tanto el extracto acuoso y el aceite de moringa oleífera (semilla), no solo tienen actividad hipoglucémica, sino también actividad protectora contra las nefronas. (p. 73)

Bamagous, y otros (Bamagous, y otros, 2018) evaluaron los mecanismos antioxidantes y antidiabéticos de la fracción de extracto de acetato de etilo de hojas de Moringa oleífera en la diabetes inducida por estreptozotocina en ratas Sprague-Dawley macho. Los hallazgos encontrados apoyan que las hojas de Moringa oleífera poseen un potente efecto terapéutico en el tratamiento de la diabetes mellitus por medio de crecientes niveles de antioxidantes e inhibición 


\section{Uso terapéutico de la moringa oleífera en pacientes diabéticos}

Vol. 3, núm. 3., (2019)

Victoria Samanta Luna Panimboza; Frowen Miguel Zambrano Solorzano; Ligia Elena Panezo

Carbo; Julian Alfonso Luna Montalván

de mediadores proinflamatorios. Razón por la cual, las propiedades antidiabéticas en la fracción de acetato de etilo de Moringa oleífera pueden ser atribuidas al rico contenido de antioxidantes y al mecanismo antiinflamatorio promovido por el extracto. Por último, estos autores recomiendan realizar más investigaciones relacionadas con los componentes fitoquímicos individuales en el extracto de acetato de etilo de la Moringa oleífera con la finalidad de usarlos como potenciales antidiabéticos y antiinflamatorios. (p. 325,326)

\section{Estudios clínicos de la Moringa Oleífera en el control de la diabetes}

Los estudios en humanos, hasta la fecha, son menores que los estudios pre clínicos y es que en materia de evidencia que respalde la acción y aplicación específica de los productos derivados de la Moringa Oleífera y su inocuidad falta camino por recorrer. No obstante, algunos estudios clínicos han evidenciado su aplicación en el tratamiento de la diabetes.

Kumari, (2010) diseñó y llevo a cabo un estudio para evidenciar clínicamente el efecto hipoglucémico de las semillas de Moringa oleífera y de Azadirachta en la diabetes mellitus tipo 2. Este autor, en una población de cincuenta y cinco (55) pacientes diabéticos, los cuales no eran dependientes de insulina (de ellos 36 hombres y 19 mujeres), en edades comprendidas entre los 30 y 60 años, del Hospital de la Universidad Acharya Nagarjuna (ANU), Guntur, dividida en dos experimentales $(n=46)$ y grupo de control $(n=9)$. Los dos primeros experimentales se administraron polvo de hojas de Moringa oleífera (8 g) y Azadirachta indica semillas en polvo (6 g) por día, respectivamente, en tres dosis divididas, durante 40 días. El tercer grupo de nueve, no recibieron ningún tratamiento y fueron designados como el grupo de control. Esta investigación reveló una disminución en los valores de glucosa en sangre, los niveles de glucosa en sangre 


\section{Uso terapéutico de la moringa oleífera en pacientes diabéticos}

Vol. 3, núm. 3., (2019)

Victoria Samanta Luna Panimboza; Frowen Miguel Zambrano Solorzano; Ligia Elena Panezo Carbo; Julian Alfonso Luna Montalván

posprandial y los lípidos sanguíneos medios, lo cual le atribuye al tratamiento el efecto antidiabético. Entre las dos hierbas seleccionadas, se encontró que el polvo de hojas de Moringa oleífera era más efectivo seguido de Azadirachta (semillas en polvo). (p. 214)

Leone, y otros, (2018) estudiaron el efecto hipoglucemiante en humanos del polvo de hojas de Moringa oleífera, evaluando la composición química del polvo de la hoja de esta planta producido en los campos de refugiados saharauis, además estudiaron su capacidad in vitro para inhibir la actividad de la $\alpha$-amilasa y su aceptabilidad sensorial en los alimentos. Asimismo, evaluaron su efecto sobre la respuesta de la glucosa posprandial. La población de estudio constó de diecisiete diabéticos saharauis y diez sujetos sanos. Estos llegaron a la conclusión en cuanto a la aceptación de su consumo habitual dentro de los alimentos que la dosis usada de 20 gr de la hoja era tolerada, sin embargo, dado su sabor amargo no resulta agradable, por tanto, recomienda realizar nuevas pruebas con dosis más bajas. En cuanto al control de la diabetes, concluyeron que el polvo de la hoja de Moringa "parece poder reducir la respuesta de la glucosa posprandial en sujetos diabéticos, y esta actividad está potencialmente relacionada, al menos en parte, con la inhibición de la actividad de la $\alpha$-amilasa". No obstante, recomiendan realizar otros ensayos clínicos que evalúen los efectos a largo plazo de este polvo en la glucosa en la sangre a los fines de demostrar los efectos hipoglucemiantes a largo plazo. (p. 11)

\section{Conclusiones.}

La medicina tradicional ha implementado en muchos lugares del mundo el uso de la Moringa Oleífera, a lo largo de los años, siendo una de las plantas medicinales con menor toxicidad comprobada en todo el mundo. No obstante, falta mucha documentación científica. Su 


\section{Uso terapéutico de la moringa oleífera en pacientes diabéticos}

Vol. 3, núm. 3., (2019)

Victoria Samanta Luna Panimboza; Frowen Miguel Zambrano Solorzano; Ligia Elena Panezo

Carbo; Julian Alfonso Luna Montalván

estudio farmacológico y químico asociados a las propiedades médicas de la planta se encuentran en etapa de desarrollo.

A pesar de que se han realizado muchos estudios preclínicos y clínicos en todo el mundo, falta mucho camino por recorrer en evidenciar sólidamente su aplicación en determinados tratamientos médicos y su inocuidad.

De los estudios revisados se concluye que los productos derivados de la planta de Moringa Oleífera (extracto de etanol de la hoja, polvo de semillas, extracto acuoso y el aceite de la semilla, extracto de acetato de etilo de hojas, entre otros), todos estos tienen efecto antidiabético, es decir, se evidencia su efectividad para el tratamiento de la diabetes principalmente por su alto contenido de antioxidantes e incluso como complemento de la dieta del paciente con esta afección. En algunos de los casos coincidieron en el aumento de peso de la población en estudio, quizás motivado a su contenido multivitamínico. En ninguno de los casos se presentaron efectos tóxicos.

Todos los estudios coincidieron en que deben realizarse mayores esfuerzos en la investigación de esta planta multifuncional para determinar sus mecanismos de acción, los principios activos que ocasionan los resultados, entre otros, a los fines de garantizar su correcta aplicación y sacar el mayor provecho de esta maravilla natural al alcance de todos los pueblos del mundo.

\section{Bibliografía.}

Al-Malki, A. L., \& El Rabey , H. (05 de Enero de 2015). El efecto antidiabético de las dosis bajas de Moringa oleifera Lam. Semillas en la diabetes inducida por estreptozotocina y 


\section{Uso terapéutico de la moringa oleífera en pacientes diabéticos}

Vol. 3, núm. 3., (2019)

Victoria Samanta Luna Panimboza; Frowen Miguel Zambrano Solorzano; Ligia Elena Panezo Carbo; Julian Alfonso Luna Montalván

nefropatía diabética en ratas masculinas. Biomed Research International, 2015, 13. $\begin{array}{lllllll}\text { Recuperado el } 02 \text { de Julio de } & 019, & \text { de }\end{array}$ https://www.ncbi.nlm.nih.gov/pmc/articles/PMC4299558/pdf/BMRI2015-381040.pdf

Bamagous, G. A., Al Ghamdi, S., Aziz Ibrahim, I., Mahfoz, A., Afify , M., Alsugoor , M., ... Rengarajan, T. (2018). Actividad antidiabética y antioxidante de la fracción de extracto de acetato de etilo de hojas de Moringa oleifera en ratas con diabetes inducida por estreptozotocina mediante la inhibición de mediadores inflamatorios. Asian Pacific Journal of Tropical Biomedicine, 8(6), 320-327. Recuperado el 01 de Julio de 2019, de http://www.apjtb.org/temp/AsianPacJTropBiomed86320-4313965_115859.pdf

Centro Nacional de Medicina Complementaria y Alternativa de los Estados Unidos. (2011). www.medlineplus.gov. Recuperado el 01 de Julio de 2019, de https://nccih.nih.gov/sites/nccam.nih.gov/files/informaciongeneral.pdf

Edoga, C., Njoku , O., Amadi, E., \& Okeke, J. (Enero de 2013). Efecto reductor del azúcar en la sangre de Moringa Oleifera Lam en ratas albinas. Revista Internacional de Ciencia y Tecnología, 3(1), 88-90. Recuperado el 05 de Julio de 2019, de https://translate.google.com/translate?hl=es-

419\&sl=en\&u=https://pdfs.semanticscholar.org/5453/ac494e374182111469e32d0803809 4125450.pdf\&prev=search

Instituto Nacional del Cáncer de los Estados Unidos. (2019). cancer.gov. (I. N. Unidos, Productor) Recuperado el 30 de Junio de 2019, de https://www.cancer.gov/espanol/publicaciones/diccionario/def/medicina-alternativa

Kumari, J. (19 de Mayo de 2010). Efecto hipoglucemiante de Moringa oleifera y Azadirachta indica en la diabetes tipo 2. Researchgate, 5(2), 211-214. Recuperado el 3 de Julio de 2019, de https://translate.google.com/translate?hl=es419\&sl=en\&u=https://www.researchgate.net/publication/266570233_Hypoglycemic_effe ct_of_Moringa_oleifera_and_Azadirachta_indica_in_type-2_diabetes\&prev=search

Leone, A., Bertoli, S., Di Lello, S., Basolli, A., Ravasenghi, S., Forlani, F., . . Battezzati, A. (09 de Octubre de 2018). Efecto del polvo de hoja de Moringa oleifera sobre la respuesta a la glucosa en sangre posprandial: estudio in vivo sobre personas saharauis que viven en campamentos de refugiados. Nutrients, 10(10), 1-12. Recuperado el 02 de Julio de 2019, de https://www.ncbi.nlm.nih.gov/pmc/articles/PMC6213450/pdf/nutrients-10-01494.pdf

Martín, C., Martin, G., García, A., Fernández, T., Hernández, E., \& Puls, J. (2013). Potenciales aplicaciones de Moringa oleifera. Una revisión. Pastos y Forrajes, 36(2), 137-149. Recuperado el 01 de Julio de 2019, de http://scielo.sld.cu/pdf/pyf/v36n2/pyf01213.pdf

Nadro, M., Audu , A., \& Glen, E. (2018). Efectos antidiabéticos del extracto acuoso y aceite de moringa Semilla de oleifera sobre las funciones hepáticas y renales en la diabetes inducida por estreptozotocina en ratas. Revista Americana de Bioquimica, 8(4), 69-74. 


\section{Uso terapéutico de la moringa oleífera en pacientes diabéticos}

Vol. 3, núm. 3., (2019)

Victoria Samanta Luna Panimboza; Frowen Miguel Zambrano Solorzano; Ligia Elena Panezo

Carbo; Julian Alfonso Luna Montalván

Recuperado el 01 de Julio de 2019, de https://translate.google.com/translate?hl=es419\&sl=en\&u=http://article.sapub.org/10.5923.j.ajb.20180804.01.html\&prev=search

Onyagbodor, O. A., \& Aprioku, J. (Octubre de 2017). El extracto de la hoja de Moringa oleifera inhibe el efecto diabetogénico de aloxan en ratas. IOSR Journal Of Pharmacywww.iosrphr.org, 7(10), 7-12. Recuperado el 01 de Julio de 2019, de https://translate.google.com/translate?hl=es-

419\&sl=en\&u=http://iosrphr.org/papers/vol7-issue10/B0710010712.pdf\&prev=search

Organización de las Naciones Unidas para la Alimentación y la Agricultura. (2019). fao.org. Recuperado el 01 de Julio de 2019, de http://www.fao.org/traditional-crops/moringa/es/

Organización Mundial de la Salud. (2016). apps.who.int. Recuperado el 01 de Julio de 2019, de https://apps.who.int/iris/bitstream/handle/10665/204877/WHO_NMH_NVI_16.3_spa.pdf ;jsessionid=E3FFFC03916E205BAF464B1B58EB968F?sequence=1

Organización Mundial de la Salud. (30 de Octubre de 2018). who.int. Recuperado el 30 de Junio de 2019, de https://www.who.int/es/news-room/fact-sheets/detail/diabetes

Ríos, R. (09 de 2017). www.eumed.net. (E. Grupo de investigación (SEJ 309) eumed.net de la Universidad de Málaga, Ed.) Obtenido de www.eumed.net: http://www.eumed.net/libros/libro.php?id=1662

Villarruel, A., López, D., Vásquez, O., Puebla, A., Torres, M., Guerrero, L., \& Nuño, K. (10 de Abril de 2018). Efecto del consumo de Moringa oleifera en ratas diabéticas. BMC Complementary \& Alternative Medicine, 18(127), 1-10. Recuperado el 01 de Julio de 2019, https://www.ncbi.nlm.nih.gov/pmc/articles/PMC5894151/pdf/12906_2018_Article_2180. pdf

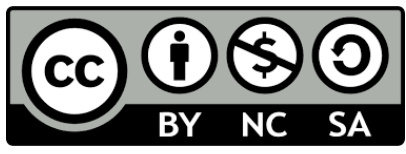

RECONOCIMIENTO-NOCOMERCIAL-COMPARTIRIGUAL

CC BY-NC-SA

ESTA LICENCIA PERMITE A OTROS ENTREMEZCLAR, AJUSTAR Y CONSTRUIR A PARTIR DE SU OBRA CON FINES NO

COMERCIALES, SIEMPRE Y CUANDO LE RECONOZCAN LA AUTORÍA Y SUS NUEVAS CREACIONES ESTÉN BAJO UNA LICENCIA CON LOS MISMOS TÉRMINOS. 